\title{
Subcellular resolution three-dimensional light-field imaging with genetically encoded voltage indicators
}

\author{
Peter Quicke, ${ }^{\text {a,b }}$ Carmel L. Howe, ${ }^{\text {a,b }}$ Pingfan Song, ${ }^{c}$ Herman V. Jadan, ${ }^{c}$ \\ Chenchen Song, ${ }^{d}$ Thomas Knöpfel, ${ }^{d, b}$ Mark Neil, ${ }^{e, b}$ Pier L. Dragotti, \\ Simon R. Schultz, ${ }^{\mathrm{a}, \mathrm{b}}, *$ and Amanda J. Foust ${ }^{\mathrm{a}, \mathrm{b}}, *$ \\ amperial College London, Department of Bioengineering, London, United Kingdom \\ ${ }^{\mathrm{b}}$ Imperial College London, Centre for Neurotechnology, London, United Kingdom \\ 'Imperial College London, Department of Electrical and Electronic Engineering, London, \\ United Kingdom \\ ${ }^{\mathrm{d}}$ Imperial College London, Department of Brain Sciences, London, United Kingdom \\ ${ }^{\mathrm{e}}$ Imperial College London, Department of Physics, London, United Kingdom
}

\begin{abstract}
Significance: Light-field microscopy (LFM) enables high signal-to-noise ratio (SNR) and light efficient volume imaging at fast frame rates. Voltage imaging with genetically encoded voltage indicators (GEVIs) stands to particularly benefit from LFM's volumetric imaging capability due to high required sampling rates and limited probe brightness and functional sensitivity.

Aim: We demonstrate subcellular resolution GEVI light-field imaging in acute mouse brain slices resolving dendritic voltage signals in three spatial dimensions.

Approach: We imaged action potential-induced fluorescence transients in mouse brain slices sparsely expressing the GEVI VSFP-Butterfly 1.2 in wide-field microscopy (WFM) and LFM modes. We compared functional signal SNR and localization between different LFM reconstruction approaches and between LFM and WFM.

Results: LFM enabled three-dimensional (3-D) localization of action potential-induced fluorescence transients in neuronal somata and dendrites. Nonregularized deconvolution decreased SNR with increased iteration number compared to synthetic refocusing but increased axial and lateral signal localization. SNR was unaffected for LFM compared to WFM.

Conclusions: LFM enables 3-D localization of fluorescence transients, therefore eliminating the need for structures to lie in a single focal plane. These results demonstrate LFM's potential for studying dendritic integration and action potential propagation in three spatial dimensions.

(C) The Authors. Published by SPIE under a Creative Commons Attribution 4.0 Unported License. Distribution or reproduction of this work in whole or in part requires full attribution of the original publication, including its DOI. [DOI: 10.1117/1.NPh.7.3.035006]
\end{abstract}

Keywords: light-field microscopy; genetically encoded voltage indicator; voltage imaging.

Paper 20044R received May 26, 2020; accepted for publication Aug. 7, 2020; published online Aug. 28, 2020.

\section{Introduction}

Cellular resolution voltage imaging enables direct observation of neuronal computation. Indeed, membrane potential imaging experiments have spatiotemporally resolved both active and passive action, and synaptic potential generation throughout dendritic and axonal arbors. ${ }^{1-14}$ Resolution of these small voltage signals at high speeds requires high photon fluxes, making wide-field single-photon (1P) imaging by far the most common voltage imaging modality. Imaging neuronal processes with this technique require the imaged membranes to lie approximately flat in the microscope's focal plane. As these experiments are typically performed in slices, the requirement for flat, healthy, and superficial cells represents a significant barrier

*Address all correspondence to Simon R. Schultz, E-mail: s.schultz@ic.ac.uk; Amanda J. Foust, E-mail: a.foust@ic.ac.uk 
to entry for experimenters. Even in the best-prepared slices, anatomy dictates that only a few cells will be oriented parallel to the surface, reducing experimental throughput, and only certain cell types feature morphology that can be well-sampled by a single plane. Iterative threedimensional (3-D) imaging in wide field via physical refocusing is often untenable due to rapid sample bleaching. Multiple approaches to improve wide-field imaging's 3-D performance have been developed. Anselmi et al. ${ }^{15}$ applied remote focusing to axially shift and tilt the wide-field focal plane as required by the sample, enabling calcium imaging along tilted dendrites. This adaptation, however, costs half of the fluorescence emission and is limited to a single tilted plane at a time. Point spread function (PSF) engineering via cubic phase masks ${ }^{16}$ or spherical aberration ${ }^{17}$ also enables parallelized volumetric sample imaging when combined with lightsheet excitation; however, to our knowledge, these approaches have not successfully been implemented to image membrane voltage.

Lack of optical sectioning with wide-field $1 \mathrm{P}$ imaging further complicates matters. Light from out-of-focus structures pollutes in-focus signals, confounding allocation of signals to axially separated processes. This issue is difficult to resolve with traditional optically sectioning confocal or two-photon microscopy approaches as they are point scanning. Sequential sampling of each pixel greatly reduces imaging bandwidth, and the fast frame rates required for voltage imaging necessitates short dwell times and, therefore, few collected photons. This restricts Poisson-noise limited signal-to-noise ratio (SNR) to low levels, making point scanning voltage imaging applicable to a limited number of experimental paradigms. ${ }^{12,18-20}$

Fluorescence excitation parallelization with multiple spots, ${ }^{21-27}$ spinning disks, ${ }^{28,29}$ blobs, ${ }^{30,31}$ lines, ${ }^{32-34}$ sheets, ${ }^{35-42}$ or specified patterns ${ }^{43-47}$ increases the photon budget, enabling functional volumetric imaging or single-plane imaging at increased speeds. A small number of these have been applied to imaging voltage in two dimensions, ${ }^{26,33,44,47}$ however, they are not able to image neuronal processes in 3D. Many of these techniques also trade-off reduced robustness to scattering compared to single-point scanning modalities for the increased excitation from parallelization.

Parallelized 3-D two-photon imaging with elongated Bessel ${ }^{48,49}$ or stereoscopic tilted ${ }^{50}$ beams excites narrow columns of fluorescence and relies on temporal and spatial sparsity of labeling and activity to demix time courses from different $z$ planes. This increases the volume rate but each columnar pixel is still addressed sequentially, limiting bandwidth. These techniques have been used to image calcium fluorescence transients but not yet voltage.

Light-field microscope (LFM) ${ }^{51}$ enables reconstruction of 3-D volumes from single twodimensional (2-D) camera images, extending wide-field imaging while maintaining its unparalleled fluorescence excitation and collection efficiency. This is achieved by inserting a microlens array (MLA) at the native image plane of the microscope and placing the image sensor at its back focal plane [Fig. 1(a)]. This disperses the angular components of the collected image [Fig. 1(b)], which can be used to infer objects' axial positions. Each LFM image consists of circular subimages [Fig. 1(c)], with each subimage resembling a pixel in an undersampled image of the scene. Within each circular subimage, each pixel location encodes a different angular sampling through the object intersecting with the subimage's location, a columnar tomographic projection through the sample. ${ }^{53}$ Light-field images are typically parameterized by the fourdimensional (4-D) function $\mathcal{L}(u, v, x, y)$, where each lenslet subimage is $\mathcal{L}(u, v, \cdot, \cdot)$ and the same specific pixel under each subimage is $\mathcal{L}(\cdot, \cdot, x, y)$. The "native LFM resolution" with which the object is laterally sampled is given by the microlens pitch divided by the objective magnification, much worse than the corresponding wide-field resolution. In exchange, the microlenses provide angular information that can be used to render views of the object from different perspectives, focus on different planes, and reconstruct 3-D volumes all from a single 2-D frame. This technique converts a key disadvantage of wide-field 1P fluorescence excitation, lack of optical sectioning, into an advantage, as out-of-focus light renders 3-D information about the sample.

Two prominent algorithms for reconstructing source volumes from LFM images have been described, namely synthetic refocusing ${ }^{51}$ and 3-D deconvolution. ${ }^{53}$ Synthetic refocusing relies on a ray optics model of LFM image formation to reconstruct images at the native LFM resolution equivalent to those of a wide-field microscope (WFM) focused at any axial plane in the sample. Focal stacks can be generated similar to standard microscope $z$-stacks by combining 

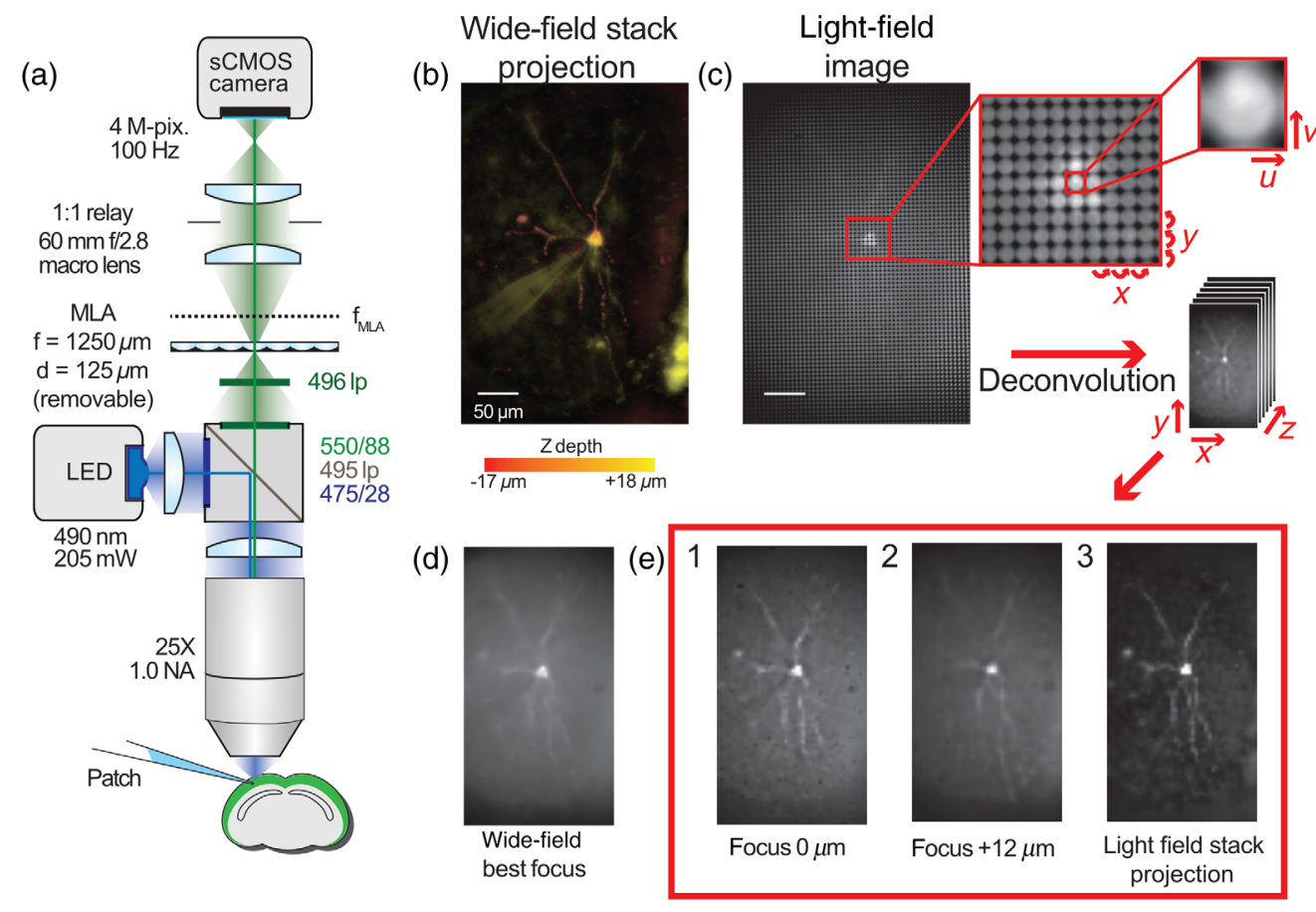

Fig. 1 Light-field microscopy enables simultaneous focusing on axially separated dendrites. (a) LFM diagram. (b) A pseudocolor $z$-projection of a wide-field image stack through a GEVI labeled cell. Depth is color coded from red (superficial) to yellow (deep). Individual dendrites follow tortuous paths in all three dimensions, so they cannot be focused on simultaneously in WFM. (c) A light-field image of the same cell showing the structure of light-field images. Each spot in the lightfield image is a spatial sampling (coordinates $x, y$ ) of the angular distribution of rays (coordinates $u, v)$ at that point. This angular and spatial information can be used to reconstruct a volume from a single image. (d) A best focus wide-field image of the single cell showing partially in-focus dendritic structures. (e) Three different images recovered from the light-field image: (e1) and (e2) are the single axial planes deconvolved showing individual dendrites seen out-of-focus in the wide-field image. (e3) A z-projection through the recovered light-field volume image showing the in-focus sections of recovered dendrites. Figure adapted from Quicke ${ }^{52}$ CC BY-SA 4.0.

images reconstructed at multiple axial depths. Each pixel in the refocused image is a weighted sum of light-field image pixels, meaning refocusing is fast. The reconstructed images, however, suffer from the same blur due to lack of optical sectioning as a standard WFM.

An alternative approach is based on reconstruction of the source volume using a forward model of light-field image formation (the LFM PSF) based on wave optics. ${ }^{53}$ Iterative deconvolution approaches, such as Richardson-Lucy $(\mathrm{RL})^{54,55}$ or the image space reconstruction algorithm (ISRA) ${ }^{56}$ find the maximum likelihood source volume given the measured image and LFM PSF in the presence of Poissonian (RL) or Gaussian (ISRA) noise. This approach can reconstruct source volumes at a lateral resolution greater than the native LFM resolution (the MLA pitch in the sample) by leveraging the fine sampling of the LFM tomographic projections. ${ }^{53}$ This increased resolution reconstruction fails where the tomographic sampling is degenerate, most notably around the native focal plane of the microscope. The source volume is also reconstructed with less axial blur than in the refocused case, increasing axial signal discriminability.

Newer light-field designs have significantly improved LFM performance and circumvented the degenerate sampling at the native focal plane. High-resolution LFM displaces the degenerate sampling away from the native focal plane, enabling higher resolution object reconstruction ${ }^{57}$. Cohen et al. ${ }^{58}$ removed the sampling degeneracy and improved the reconstruction via placing a phase mask in the objective back focal plane. Particularly important, Fourier $\mathrm{LFM}^{59,60}$ modifies the LFM design to place the MLA in the Fourier plane of the microscope. This design eases reconstruction computational cost due to its laterally invariant PSF and also does not suffer from 
reconstruction issues around the native focal plane. However, the PSF periodicity does result in reconstruction artifacts necessitating a reduction in the field of view (FOV) to remove. Recently, Liu et al. ${ }^{61}$ further improved on LFM with a particularly promising design, removing reconstruction artifacts using a random MLA, and increasing the reconstructable depth of field using microlenses of different focal lengths.

Electrical length constants in neurons are on the scale of tens to hundreds of microns, making increased lateral pixel size less disadvantageous for voltage imaging. Over-resolving electrical fluctuations by imaging at or below the diffraction limit are typically unnecessary and can even hurt SNR by increasing the relative impact of non-Poisson noise, such as read noise. Spatial resolution is therefore often sacrificed in voltage imaging experiments to increase speed or SNR. Many such experiments use low read noise, high sensitivity CCD sensors featuring low pixel counts, with pixels often measuring several microns across in the sample plane. Even with higher pixel count detectors, the relatively low sensitivity of many voltage probes means multiple pixels are often binned to increase SNR to acceptable levels. Therefore, LFM's decreased native lateral sampling rate suits voltage imaging well, and deconvolution of LFM voltage imaging time series can be implemented without oversampling to reduce computational cost.

LFM has successfully imaged calcium over large volumes in C. elegans and zebrafish, ${ }^{62,63}$ and in both head-fixed and behaving mice. ${ }^{64-66}$ Voltage dynamics have also been imaged successfully without single-cell resolution in Drosophila ${ }^{67}$ and larval zebrafish ${ }^{60}$ as part of wholebrain imaging setups alongside calcium imaging. LFM has not, to our knowledge, been applied to studying subcellular or single-cell resolution voltage dynamics in any sample, despite its apparent suitability. In this study, we apply LFM to subcellular genetically encoded voltage indicator (GEVI) imaging in acute mouse brain slices. We combine this technique with a recently reported transgenic strategy driving sparse expression in a random subset of layer $2 / 3$ cortical pyramidal neurons, which enables the resolution of single-cell level voltage signals in neuronal somata and dendrites. ${ }^{68,69}$

We demonstrate that LFM can simultaneously image axially separated dendrites, enabling single-shot capture and localization of GEVI fluorescence transients in the 3-D dendritic arbor. We compare and evaluate deconvolution and synthetic refocusing for different GEVI imaging applications, while using a coarse deconvolution approach with no lateral oversampling to reduce computational cost. We also apply a recently developed LFM PSF calculation ${ }^{70}$ for highNA objectives. We show that LFM enables 3-D localization of dendritic and somatic GEVI fluorescence transients and compare the extent to which refocused and deconvolved light fields enable lateral and axial transient localization. Finally, we compare temporal signal SNR between LFM and WFM.

\section{Methods}

This section reproduces methods described by Quicke. ${ }^{52}$ We designed our LFM following the principles set out by Levoy et al. ${ }^{51}$ We adapted a wide-field imaging system by placing an MLA at the microscope image plane and used a 1:1 relay lens (Nikon 60-mm $f / 2.8$ D AF Micro Nikkor Lens) system to image the MLA back focal plane onto our camera chip [ORCA Flash 4 V2, $2048 \times 2048$ pixels, 6.5- $\mu \mathrm{m}$ pixel size, Hamamatsu, see Fig. 1(a)]. The lateral resolution is given by the MLA pitch divided by the magnification of the objective. Using our $25 \times$ objective (1.0 NA, XLPLN25XSVMP, Olympus), we chose our system to have 5- $\mu \mathrm{m}$ lateral pixels, dictating a microlens pitch of $125 \mu \mathrm{m}$.

The axial resolution is defined by the number of resolvable diffraction-limited spots behind each microlens. ${ }^{51}$ Assuming a central emission wavelength of $550 \mathrm{~nm}$ for mCitrine, the förster resonance energy transfer (FRET) donor in VSFP-Butterfly $1.2,{ }^{71}$ the spot size in the camera plane is $6.46 \mu \mathrm{m}$ using the Sparrow criterion. With a $125-\mu \mathrm{m}$ pitch MLA, we can resolve $N_{u}=$ 19 distinct spots under each microlens. The axial resolution when synthetically refocusing our LFM can, therefore, be calculated as $7.81 \mu \mathrm{m} .^{51}$

To efficiently use the camera sensor, the exit pupil of the objective should map through the MLA to produce circles on the light-field plane that are just touching, requiring that the objective image-side $f$-number $(f / 12.5)$ equal the MLA $f$-number. We chose an $f / 10$ MLA (MLA-S125- 
f10, RPC Photonics), an off-the-shelf part that came close to matching while being a larger aperture.

\subsection{Imaging}

This study was carried out in accordance with the recommendations of the UK Animals (Scientific Procedures) Act 1986 under Home Office Project and Personal Licenses (project licenses 70/7818 and 70/9095). Slices were made from four mice aged 31, 32, 32, and 175 days transgenically modified to sparsely express VSFP-Butterfly $1.2^{71}$ using the method previously described by Quicke et al. ${ }^{68}$ and Song et al. ${ }^{69}$ These transgenic mice express the GEVI in cortical layer $2 / 3$ pyramidal neurons under the intersectional control of TetO and destabilized Crerecombinase. ${ }^{72-74}$ The destabilized Cre-recombinase was stochastically restabilized to induce sparse expression of the voltage indicator via two IP injections of a total of $2 \times 10^{-4} \mathrm{mg} \mathrm{kg}^{-1}$ Trimethoprim (TMP, Sigma) over two consecutive days as described by Song et al. ${ }^{69}$

Slices were prepared at least 2 weeks post-TMP injection using a method adapted from Ting et al. $^{75}$ (the "protective recovery" method ${ }^{76}$ ). Slices of $400 \mu \mathrm{m}$ were cut with a Camden Microtome 7000 in ice cold 95\% $\mathrm{O}_{2} / 5 \% \mathrm{CO}_{2}$ oxygenated artificial cerebrospinal fluid (ACSF) containing: (in mM) $125 \mathrm{NaCl}, 25 \mathrm{NaHCO}_{3}, 20$ glucose $2.5 \mathrm{KCl}, 1.25 \mathrm{NaH}_{2} \mathrm{PO}_{4}$, $2 \mathrm{MgCl}_{2}$, and $2 \mathrm{CaCl}_{2}$. The slices were then immediately transferred into NMDG-ACSF ${ }^{75}$ containing: (in mM) $110 \mathrm{~N}$-methyl-D-glucamine, $2.5 \mathrm{KCl}, 1.2 \mathrm{NaH}_{2} \mathrm{PO}_{4}, 25 \mathrm{NaHCO}_{3}, 25$ glucose, $10 \mathrm{MgCl}_{2}, 0.5 \mathrm{CaCl}_{2}$, adjusted to 300 to $310 \mathrm{mOsm} / \mathrm{Kg}, \mathrm{pH} 7.3$ to 7.4 with $\mathrm{HCl}$ and oxygenated with $95 \% \mathrm{O}_{2} / 5 \% \mathrm{CO}_{2}$ at $36^{\circ} \mathrm{C}$ for $12 \mathrm{~min}$ before being transferred back into the original sodiumcontaining ACSF for at least an hour before patching and imaging.

Fluorescent cells were patched under oblique infrared illumination $(780 \mathrm{~nm})$ with pipettes of resistances between 3 and $10 \mathrm{Mohms}$ when filled with intracellular solution containing: (in $\mathrm{mM}) 130 \mathrm{~K}$-gluconate, $7 \mathrm{KCl}, 4$ ATP-Mg, 0.3 GTP-Na, 10 phosphocreatine-Na, and $10 \mathrm{HEPES}$ is 4-(2-hydroxyethyl)-1-piperazineethanesulfonic acid. We digitized current clamp signals (Power 1401 digitizer; Cambridge Electronic Design) from a Multiclamp 700B amplifier (Axon Instruments). At room temperature, we imaged at 100 frames/second for $2.5 \mathrm{~s}$ while injecting current pulses lasting 50 and $100 \mathrm{~ms}$. A 100-Hz imaging is sufficient for detecting action potentials with this voltage indicator due to the indicator's slow kinetics. This GEVI, unlike recently developed indicators or organic voltage dyes, reports a low-pass filtered version of the membrane voltage signal. Second, as discussed by Quicke et al. ${ }^{68}$ lower frame rates can be useful for lowSNR spike detection, as the signal is further low-pass filtered by the camera integration period, and the longer frame period allows collection of more photons, increasing shot-noise limited SNR. Higher frame rates would better resolve action potential timing and kinetics. Each pulse elicited depolarization to threshold evoking a single action potential or burst of two to three action potentials. We powered a 490-nm LED (M490L4, Thorlabs) with a constant current source (Keithley Sourcemeter 1401) to illuminate the sample at 3 to $11 \mathrm{~mW} / \mathrm{mm}^{2}$. Sets of light-field time series and wide-field time series acquisitions were interleaved by removing and replacing the MLA by hand. We averaged between 4 and 16 sweeps per imaging condition. The LED was collimated with an $f=16-\mathrm{mm}$ aspheric lens (ACL25416U0-A, Thorlabs) and filtered with a 475/28 nm excitation filter (FITC-EX01-CLIN-25, Semrock). Fluorescence was collected using a 495-nm long-pass dichroic (FF495-Di03, Semrock) along with a 550/88-nm collection filter (FF01-550/88, Semrock) and 496 long-pass filters (Semrock FF01-496/LP) to attenuate any excitation light transmitted by the dichroic. Imaging data were acquired with Micromanager. ${ }^{77}$ Imaged cells' somata lay between 11 and $40 \mu \mathrm{m}$ below the slice surface, with a median depth of $29 \mu \mathrm{m}$. These cells all lie within the photon mean free path of the slice surface, ${ }^{78}$ and our reconstruction methods do not take tissue scattering into account. Data were analyzed with custom Python scripts using SciPy packages. ${ }^{79}$

\subsection{Light-Field Reconstruction}

We reconstructed source volumes using two techniques to compare their performance for singlecell voltage data. We calculated $(x, y, z, t)$ volume time series using synthetic refocusing ${ }^{51}$ and ISRA ${ }^{56,62}$ using a PSF calculated using the method described in the section as follows. 
RL deconvolution ${ }^{53-55}$ was also tested on the data, however, little discernible difference in the results was observed.

\subsubsection{Light-field PSF calculation}

We calculated LFM PSFs differently to previously described,${ }^{53}$ using the method described by Quicke ${ }^{52}$ and Quicke et al. ${ }^{70}$ Briefly, to calculate the field at the MLA, we considered how a highNA objective lens collects the field from an oscillating electric dipole at position $r$ near the microscope focus, $|r| \ll f$, at the origin, calculating the Fourier transform of the field in the objective back focal plane. We assumed that we could model the behavior of a point source consisting of randomly oriented fluorescent molecules as the incoherent sum of dipoles along three orthogonal directions. We then used the same method as described by Broxton et al..$^{53}$ to model transmission through the MLA and to the camera.

We calculated the PSF for GEVI imaging deconvolution for 550-nm emission. We did not oversample the deconvolution as resolving voltage signals generally requires averaging pixels to approximately the native LFM resolution. We, therefore, generated a single light-field kernel for each depth by averaging over kernels sampled for point sources at different lateral positions under the microlens, weighting each point in the average by a 2-D Hamming window function of a width equal to our microlens' pitch. We averaged over kernels sampled at five times finer than the native microlens resolution. The ISRA was used to deconvolve the data.

\subsubsection{Volume reconstructions}

Having obtained our downsampled PSF, we deconvolved our volume using a similar procedure to previous studies. A key difference is that only a single 2-D convolution was required for each depth in the reconstructed volume for the forward and backward projections, respectively, as we did not increase the lateral sampling rate. We applied the deconvolution scheme independently to each frame of the image time sequences, using a cluster to parallelize the data processing. Deconvolution of a single frame took around 30 to $40 \mathrm{~min}$ for a 21 iteration deconvolution of $21 z$ planes on a single CPU. We employed a large cluster to process the individual frames simultaneously, enabling 5000 frames to be processed overnight. We did not use a parallel algorithm within each deconvolution to leverage, e.g., GPU processing, as the computing resources available to us were better suited to data parallelism. As with previous studies, this would greatly increase the rate of individual frames, although it would also likely reduce the number of simultaneous frames that could be deconvolved for typical cluster setups.

Synthetic refocusing, based on a ray optics model of light-field image formation, is a simpler approach to volume reconstruction that is also much less computationally intensive. Images focused at different $z$-depths can be constructed by combining individual perspective views using the formula derived by $\mathrm{Ng}$ et al.$^{80}$ Linear interpolation in this summation results in each pixel being the weighted sum of pixels of the original light-field image. This reconstruction is much faster than the iterative deconvolution methods and also does not suffer from noise amplification. ${ }^{81}$

\subsection{Volume Time Series Analysis}

\subsubsection{Effect of reconstruction on SNR}

To compare the effect of different reconstruction techniques on voltage signal SNR, we reconstructed single planes from volumes at the LFM focus. We compared synthetically refocused time series with time series deconvolved using ISRA for different iteration numbers. Regions of interest (ROIs) were manually chosen over the soma and its surround and were identical for the synthetically refocused and deconvolved volumes.

As we were collecting fluorescence from the VSFP-Butterfly 1.2 FRET donor, fluorescence decreased upon membrane depolarization. ${ }^{71}$ Therefore, the traces shown Figs. 2 and 3 are inverted. To measure SNR, we calculated the signal as the fifth percentile value during a stimulus and relaxation period of $200 \mathrm{~ms}$ with the median value of the $100 \mathrm{~ms}$ before the stimulation 


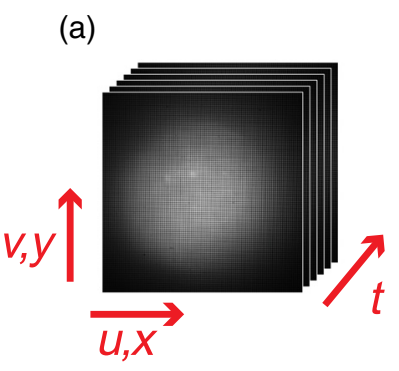

(b)

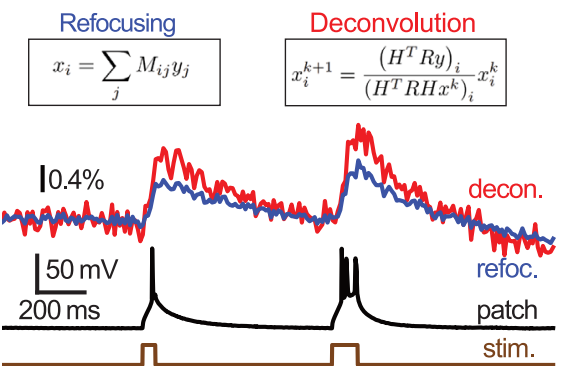

(c)

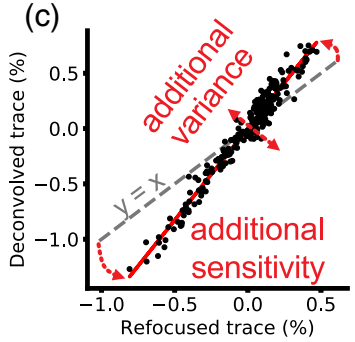

(e)

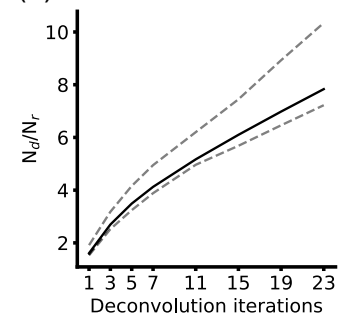

(d)

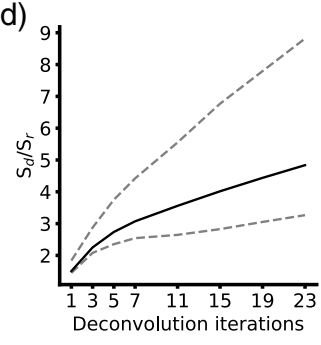

(f)

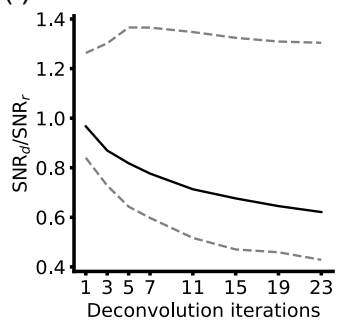

Fig. 2 Comparison of different reconstruction methods on SNR. (a) Light-field time series were collected of functional voltage signals from sparsely expressed GEVIs. (b) Time series were extracted from in-focus image sequences of the soma via refocusing (left) and ISRA deconvolution (right) and the signal and noise were compared. (c) Deconvolved and refocused signals are strongly linearly correlated, as can be seen from plotting the individual trace time points. The additional noise variance due to deconvolution can be identified as the residual from the linear fit. The increased signal level can be seen as the increased fit gradient over unit slope (gray dashed line). Both the (d) noise and (e) signal increase monotonically with increasing deconvolution iteration, leading to an overall reduction in (f) SNR with iteration number. At low iteration number, deconvolution and refocusing are very similar. At large iteration number, the SNR is decreased relative to refocused; however, increased axial sectioning may still motivate the use of deconvolution methods. Solid lines are median of $n=15$ cells and dashed lines indicate 25th and 75th percentile values. Traces in (b) were generated from an average of eight sweeps.

period subtracted. The noise level was calculated as the standard deviation of a 350-ms period during no intracellular current stimulus.

\subsubsection{Depth-time plots}

To determine the center of mass of the signal for different cellular ROIs, we extracted time courses from each refocused or deconvolved depth, then filtered the resulting depth-time 2-D arrays with a median filter of 11 samples in the time axis $(110 \mathrm{~ms})$ and three samples in the $z$ axis $(15 \mu \mathrm{m})$.

\subsubsection{Comparison of light-field and wide-field SNR}

We compared the SNR between trials of the same cell for image sequences taken with wide-field and light-field imaging systems. We compared the SNR between refocused and wide-field images for the same number of repeats using ROIs calculated to be the same for both imaging 

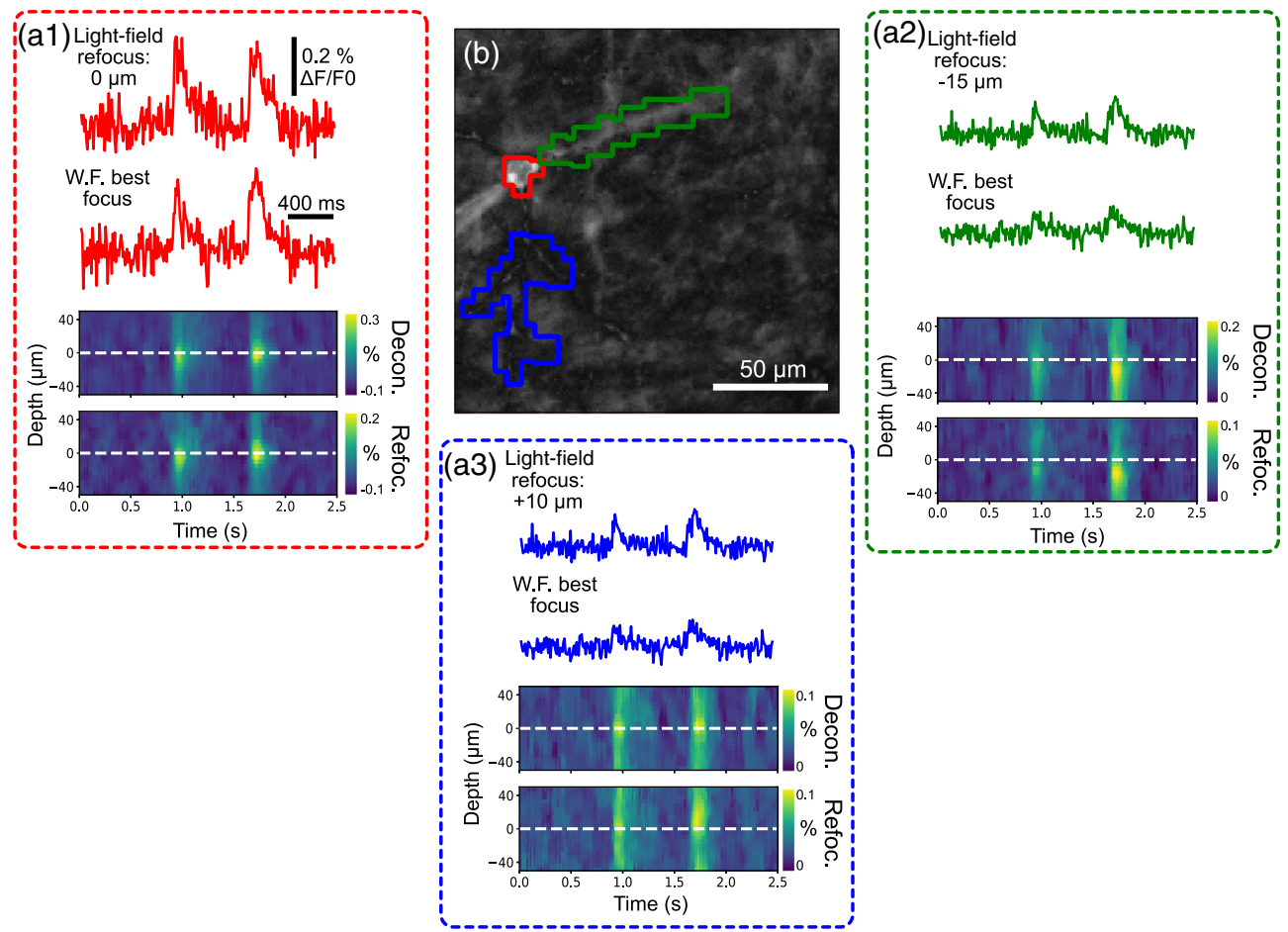

(c1)

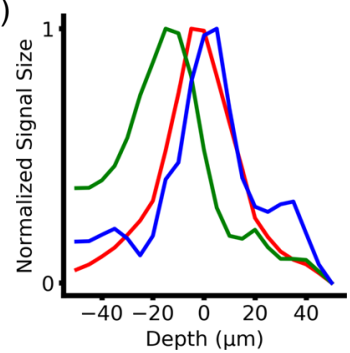

(c2)

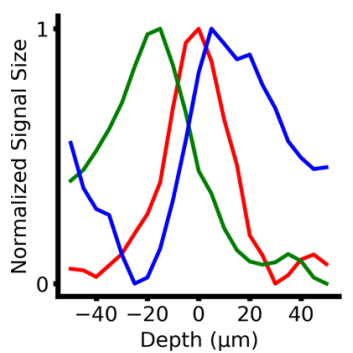

Fig. 3 Deconvolution LFM resolves 3-D localized voltage signals. (a1)-(a3) Time courses and depth-time plots showing signals from different cellular compartments [shown in (b)] localized at different depths. (a1) The somatic signal is maximal in the wide-field and native light-field focal planes, while (a2) the apical dendrite descends into the slice with its ROI localized $15 \mu \mathrm{m}$ deeper. The signal from (a3) a basal dendrite is superficial to the soma, and its best focal depth is difficult to localize due to the broad axial extent of the refocused signal. The basal and apical dendritic fluorescence transients in the wide-field time courses have smaller signals than the light-field signals as they are out-of-plane when focused on the soma. (c) The normalized signal size for each ROI across different (c1) deconvolved and (c2) refocused depths. Deconvolution increases the axial localization of signals. The data are an average of eight sweeps.

modalities. For 8/12 cells, an extra aperture was introduced into our LFM to compensate for chromatic aberration, reducing the light throughput of the microscope by between $1 / 2$ and 3/4 during light-field imaging compared to the equivalent wide-field trials. To account for this, the SNR for these trials was adjusted by a factor equal to the square root of the ratio of the mean brightness of the first imaging trials from the light-field and wide-field trials. The microscope was realigned to account for chromatic aberration before the final $4 / 12$ cells, which means that the design light throughput of the microscope was the same between light-field and wide-field trials. For these trials, the raw SNR was included in the analysis.

\subsubsection{Signal spread analysis}

We compared the lateral and axial signal spread using a method similar to our previous work. ${ }^{68}$ We quantified the neuronal voltage signal strength in each pixel to create 2-D or 3-D "activation 
maps" by calculating the temporal correlation coefficient of each pixel's time course with a seed time course from the somatic ROI.

We compared the spatial autocorrelations of these activation maps to quantify the average signal crosstalk between cellular voltage signals. ${ }^{68}$ In our previous work, we described how the autocorrelation can be used to quantify the average signal power a cell contributes to a specific pixel's time course and quantified this effect for this preparation with WFM in two dimensions. ${ }^{68}$ In this work, we calculated the 3-D autocorrelations of the light-field volumes and 2-D autocorrelations of the wide-field volumes using Fast Fourier transform (FFT)-based convolution, setting the central $10 \times 10$ pixels of the autocorrelations to the mean of their perimeter to remove a central noise peak.

\section{Results}

\subsection{Light-Field Microscopy Enables Simultaneous Imaging of Axially Separated Dendrites.}

We demonstrated LFM's ability to resolve axially separated structures by imaging a cell with a complex 3-D dendritic arbor using both WFM [Fig. 1(b)] and LFM [Fig. 1(c)]. No single plane wide-field image was able to simultaneously bring all the dendrites into a good focus [Fig. 1(d)]; however; in different planes from a volume reconstructed by deconvolution different dendritic structures could be clearly distinguished [Figs. 1(e1) and 1(e2)]. The same cellular features can clearly be seen in a standard deviation projection through the reconstructed LFM stack [Fig. 1(e3)] and a wide-field $z$ stack through the same cell [Fig. 1(b)], both projections through stacks at $1-\mu \mathrm{m}$ axial increments].

\subsection{Comparison of the Effect of Different Reconstruction Methods on Signal-to-Noise Ratio}

Low-sensitivity GEVIs mean SNR is of utmost importance in voltage imaging analysis strategies, and so we first compared the performance of the deconvolution and refocusing reconstruction approaches on this metric. We reconstructed the volume time series for 15 cells from light field (LF) time series [Fig. 2(a)] and extracted optical time courses from ROIs over the individual cell's soma at the native focal plane and compared the SNR between deconvolved and refocused volumes [Fig. 2(b)]. Commonly used LFM iterative reconstruction schemes are prone to noise amplification $^{82}$ which increases with iteration number. It is therefore crucial to understand when to stop the iteration scheme. Early stopping provides a regularizing effect on the deconvolution scheme, reducing noise contamination in the final reconstruction at the cost of model fidelity. ${ }^{83}$ We used the refocused images as a baseline comparison for the iteration analysis due to the ease of their reconstruction. We found that for all iteration numbers, the noise and signal level were increased by deconvolution which increased sensitivity and variance [Figs. 2(c)-2(e)]. The signal significantly increased from $0.3 \%(0.2 \%, 0.4 \%)$ [all results presented as median interquartile range (IQR)] in the refocused time series to $1.4 \%(0.9 \%, 1.7 \%)$ for the 21 iteration deconvolved traces (Wilcoxon signed rank, $n=15, z=0.0$, and $p=0.0003$ ). The noise significantly increased from $0.05 \%(0.04 \%, 0.08 \%)$ in the refocused time series to $0.4 \%(0.3 \%, 0.5 \%)$ for the 21 iteration deconvolved traces (Wilcoxon signed rank, $n=15, z=0.0$, and $p=0.0002$ ). This resulted in the SNR reducing from approximately the same as the refocused $[1.0(0.8,1.3)]$ case for a single deconvolution iteration to around half that of the refocused case $[0.6,(0.4,1.3)]$. We also processed the light-field time series using RL deconvolution and found no substantial differences compared to ISRA.

\subsection{Light-Field Microscopy Resolves 3-D Localized and Axially Separated Voltage Signals}

We then explored a key advantage of subcellular resolution light-field voltage imaging: 3-D imaging of neuronal processes. Achieving this requires signals from different planes to be 
discriminable in volume reconstructions. Axial discriminability depends on intrinsic factors, such as axial resolution, and also extrinsic factors, such as cellular morphology and signal spread due to tissue scattering. To demonstrate the resolution of subcellular voltage transients in 3D, we reconstructed 4-D $(x, y, z, t)$ volumes from light-field image time series and compared the temporal signals from ROIs over different dendritic and somatic structures in multiple axial planes.

Figure 3 demonstrates LF imaging's ability to axially localize functional voltage signals from neuronal processes and thereby image functional activity in 3D with SNR unachievable by any equivalent wide-field system. Figures 3(a1)-3(a3) show single plane and multiplane time courses from three different ROIs over cellular compartments from a neuron distributed over multiple axial planes. A somatic ROI [Fig. 3(a1)] in the native focal plane of both wide-field and light-field images contains action potential evoked fluorescence transients approximately equal in signal size for both light field and wide field (top). The depth-time plots show the functional signal localized to the native focal plane in the LF functional stacks (bottom). In contrast, an ROI over the apical dendrite [Fig. 3(a2)] has the largest signal when the LF image is refocused $15 \mu \mathrm{m}$ deeper into the slice, and the signal in the equivalent wide-field ROI is much smaller. The depth-time plots for this ROI from both deconvolved and refocused stacks also clearly show the center of mass of the signal located deeper than the native focal plane [Figs. 3(c)1 and 3(c)2, bottom]. Signals from a basal dendrite [Fig. 3(a3)] are similarly larger in the LF image refocused $10 \mu \mathrm{m}$ shallower than the native focal plane. The corresponding depth-time plots show a slight shift in the signal center of mass to a shallower depth, especially in the refocused case.

Plots of signal size as a function of depth for the refocused and deconvolved cases [Figs. 3(c1) and 3(c2)] show the axial localization as distinctly different planes for each ROI and also demonstrate a key advantage of deconvolved over refocused reconstructions: increased accuracy in axial localization of functional signals.

\subsection{Deconvolution Increases Axial Localization of Functional Voltage Signals}

The transients from refocused volumes exhibit a larger axial PSF width compared to the deconvolved traces [Fig. 3(c)]. Hence, these signals are smeared out, reducing distinguishability of signal contributions from different planes. To quantify this effect, we generated volumes showing the distribution of functional signal. We generated a time course from an in-focus somatic ROI and calculated the temporal correlation coefficient of every pixel in the volume for refocused and deconvolved volume time series. Pixels with high correlation coefficients are interpreted as having a large response to the intracellular current injection, and so a volume map of these reveals morphology of structures through which the functional signal propagates. These activation maps of voltage signal (Fig. 4) enabled us to quantify the spatial signal distribution from the same cells with different imaging techniques. Activation maps from wide-field imaging trials show blurring around the soma from out-of-focus basal dendrites [Fig. 4(a)]. Comparatively, $z$ projections from a $70-\mu \mathrm{m}$ region around the soma generated from the deconvolved activation volume [Fig. 4(b)] reveals the structures that cause this blur. A projection through $70 \mu \mathrm{m}$ around the focus from the refocused case shows significantly more blurring due to the poor axial sectioning of this technique [Fig. 4(c)]. We used the 3-D autocorrelation of these activation maps to quantify the spread of the signal in 3D (see Sec. 2.3.4). Measuring the autocorrelation of the activation maps provides a convenient method for comparing the average voltage signal lateral and axial width between reconstruction approaches, as complex structures distributed in 3D are automatically "aligned." The autocorrelation of the cellular signal can also be used to quantify the contribution of signal crosstalk from an adjacent cell to nearby pixels. ${ }^{68}$ To this end, we first quantified how the peak autocorrelation from each cell, and therefore functional signal contribution, decayed axially. Axial smearing can be seen in reconstructions from both deconvolution and refocusing [Fig. 4(d)], although the effect is much more severe in refocused traces. The smearing appears in the axial autocorrelation as both broadening of the central peak and increased side lobes [Fig. 4(e)]. The central peak width and side lobes decrease with increased deconvolution iteration number [Figs. 4(f) and 4(g)], thus increasing the axial sectioning. 
(a)

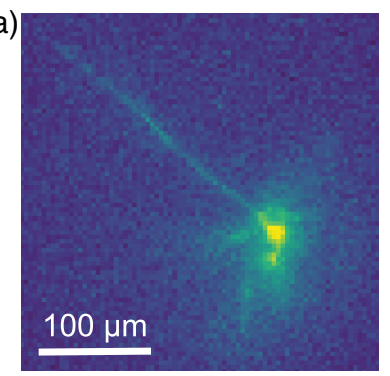

(b)

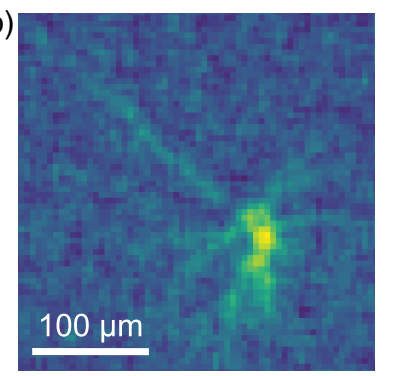

(c)

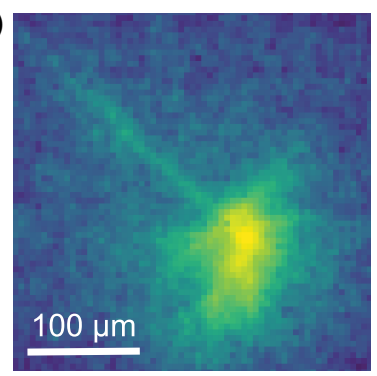

(d1)

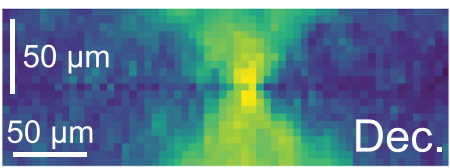

(e1)

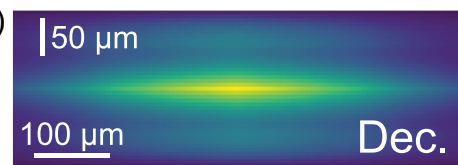

(d2)

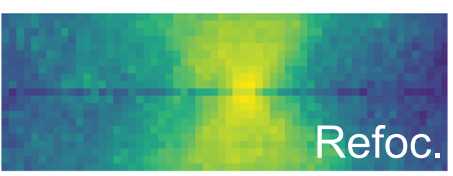

(e2)
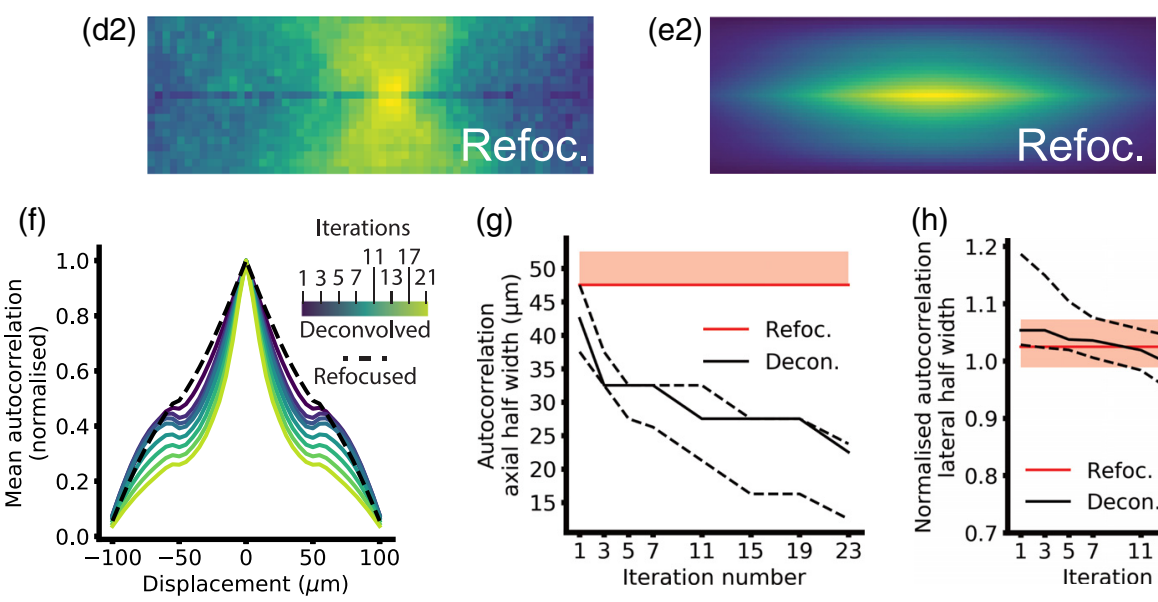

(g)

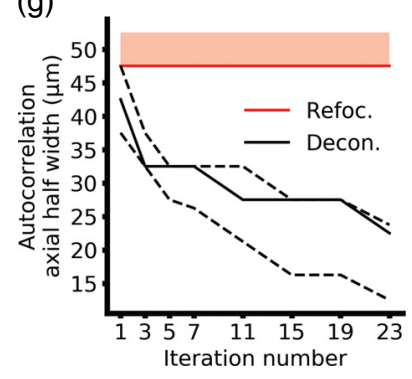

(h)

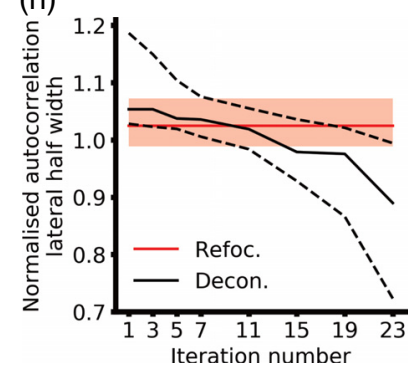

Fig. 4 Mapping dendritic signals. (a) Wide-field "activation" image. Yellow pixels contain large voltage signals, while blue pixels contain low or no voltage signals. (b) Deconvolved activation image, sum projection from -35 to $+35 \mu \mathrm{m}$, five deconvolution iterations. (c) Refocused activation image, sum projection from -35 to $+35 \mu \mathrm{m}$. (d) $x z$ maximum intensity projections through (d1) deconvolved, and (d2) refocused activation images showing the different axial sectioning. (e) Mean $x z$ projections through the autocorrelations. (f) Normalized maximum autocorrelation for different depths from refocused and deconvolved LFM activation volumes. The secondary peaks arise from the elongated axial PSF, and these can be seen decreasing as the iteration number increases. (g) Median autocorrelation axial half widths for $n=12$ cells with iteration number. Dashed lines represent quartile values. Red line is the refocused median width and shaded area of the refocused IQR. (h) Median autocorrelation lateral widths normalized to wide-field lateral widths for refocused images (red and shaded area IQR) and different deconvolution iterations (black lines and dashed lines IQR).

The autocorrelation widths decreased significantly from 1 to 21 iterations [median dropped from $42.5(37.5,47.5)$ to $22.5(12.5,23.75) \mu \mathrm{m}, z=0$, and $p=0.002]$, and for both cases, the axial spread was significantly lower than refocused [median of $47.5(47.5,52.5) \mu \mathrm{m}, p=0.001$, and $p=0.002$ for 1 and 21 iterations, respectively]. Significance tests were performed with a Friedman $\chi^{2}$ with post hoc Bonferroni-corrected Wilcoxon signed-rank tests (significant at $p<0.017$ ). Here, $n=12$ cells from 12 slices from four mice. Friedman $\chi^{2}=24$ and $p=6 \times 10^{-6}$.

Finally, we compared how deconvolution and refocusing affected lateral signal localization compared to the equivalent wide-field time series [Fig. 4(h)]. We measured the width of radially averaged autocorrelations normalized to matched wide-field trials for the refocused and deconvolved cases. We found that the lateral signal spread significantly decreased from 1 to 21 iterations [median dropped from $1.05(1.03,1.18)$ times larger than wide-field trials to $0.89(0.73,0.99)$ times larger, $Z=0, p=0.002]$, from significantly larger than the wide field and refocused at 1 iteration 


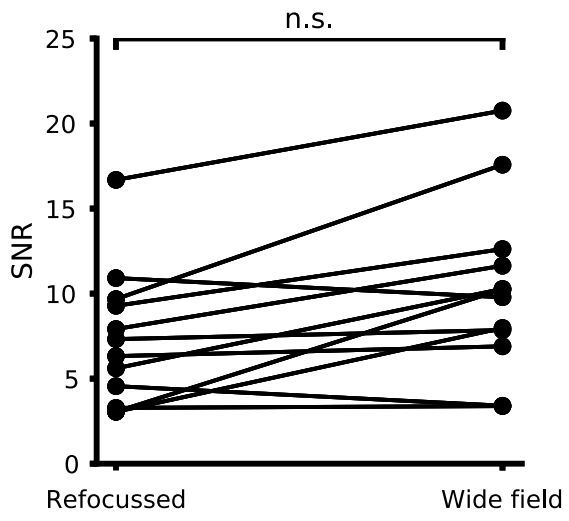

Fig. 5 Comparison of light-field SNR and wide-field SNR. Points correspond to mean SNR between paired light-field and wide-field trials. LFM SNR does not differ significantly from wide field. For $8 / 12$ trials, we included a correction factor due to a misalignment in the LFM as discussed in Sec. 2.3.3.

( $z=1, p=0.003$ and $z=0, p=0.002$, respectively), to significantly smaller than the refocused at 21 iterations $(z=0.0, p=0.003)$. The refocused widths did not differ significantly from the matched wide-field trials [median $1.02(0.99,1.07)$ times larger, $z=17$, and $p=0.08$ ]. Significance tests were performed with a Friedman $\chi^{2}$ with post hoc Bonferroni-corrected Wilcoxon signed-rank tests on the raw widths (significant at $p<0.0083$ ). Here, $n=12$ cells from 12 slices from four mice. Friedman $\chi^{2}=26, p=9 \times 10^{-6}$ (Fig. 5).

In total, our analyses reveal that deconvolution improves both axial and lateral signal localization, but decreases temporal signal SNR compared to synthetic refocusing, with both effects intensifying with increasing iteration number.

\subsection{Temporal signal SNR is Unaffected by Light-Field Imaging}

We measured the SNR for paired wide-field and refocused light-field imaging trials in the same cells. For 8/12 trials, we included a correction factor due to a misalignment in the LFM as discussed in Sec. 2.3.3. The SNR did not change significantly between the light-field and wide-field cases (Wilcoxon signed-rank test, $n=12$ cells from 12 slices and four mice, $z=32, p=0.6$ ), with a median light-field SNR of $8.4(5.2,11.4)$ and a median wide-field SNR of $10.0(7.6,11.9)$ (Fig. 5). We measured the SNR from equivalent pixel areas in wide-field and light-field image series to compare the two techniques. This potentially advantages light field over wide field, as more intricate ROIs can be used with the smaller wide-field pixels, reducing signal spatial averaging and increasing signal size. We expect this effect to be small for the somatic ROIs we used, as the pixels external to the ROIs typically still contained scattered or dendritic voltage signals, and so the increased granularity of the wide-field ROIs would not provide a large SNR advantage. Furthermore, this disadvantage is greatly ameliorated in more recent LFM implementations, ${ }^{57,59,61}$ which typically have better lateral resolution, especially at the LFM focal plane.

\section{Discussion}

We have shown that LFM enables 3-D subcellular GEVI imaging of somatic and dendritic structures. We demonstrated that LFM enables simultaneous imaging of axially separated dendrites, overcoming a key limitation of wide-field imaging. We further showed that functional voltage signals from dendrites could be axially resolved at different depths. This finding is the key to demonstrating LFM's utility for studies of dendritic integration or synaptic mapping.

We compared how synthetic refocusing and deconvolution-based reconstruction techniques perform with respect to spatial signal localization and temporal SNR. Synthetic refocusing is computationally simple and can be used to process light fields online, during an experiment, or post hoc. Refocusing features have better temporal signal SNR but poorer lateral and axial 
confinement compared to deconvolution. Deconvolution has two major disadvantages: computational cost and noise amplification. As the LFM PSF is not shift invariant, it is described by a five-dimensional matrix, complicating reconstruction. The periodicity it displays under lateral shifts by integer multiples of the microlens pitch, however, enable deconvolution to be performed efficiently using FFT-based convolutions. Despite this, even small increases in lateral sampling in the deconvolved volume increase the computational cost of reconstruction drastically. Reconstructing $n_{z} z$ planes in a volume with a lateral increase in sampling over the native LFM sampling of $m$ requires $2 \times n_{z} \times m^{2}$ 2-D convolutions per iteration, precluding online image processing. Second, both RL and ISRA tend to amplify noise in their outputs due to their lack of regularization. ${ }^{82}$ This noise may be acceptable when imaging high-SNR calcium signals, however, it can dominate small, dim voltage signals. Incorporating regularization into the deconvolution approaches to suppress noise overfitting could also ameliorate deconvolution's effects on temporal SNR. The iteration analysis described in Secs. 3.2 and 3.4 should aid future studies using early stopping of the deconvolution scheme as implicit regularization, as they provide a quantitative measure of the gains and losses at different regularization levels.

For this study, we used the original LFM design, which has since been improved upon significantly by multiple groups. ${ }^{57-61}$ These advances variously improve the spatial resolution and decrease the computational cost of deconvolution; however, the basic image formation concept remains the same in all the new modalities. The results in this study are likely applicable to voltage imaging with these newer light-field modalities as the reconstruction approaches are similar. In this study, we imaged VSFP-Butterfly 1.2, an older generation probe. GEVI technology has advanced dramatically recently, greatly increasing their sensitivity, and with these new sensors, noise amplification due to deconvolution in the light-field volume reconstruction may become less significant. Although VSFP-Butterfly 1.2 exhibits lower sensitivity than several recently reported probes, ${ }^{20,84-88}$ we were able to express it sparsely and strongly to enable single-cell GEVI imaging without somatic restriction, which would preclude study of subcellular signals. ${ }^{68,69}$ The slow kinetics of the probe used in this study also enabled resolution of action potentials at 100 frames/s without severe aliasing. Although we could resolve single-sweep signals, signal averaging was required to resolve smaller dendritic signals with adequate SNR. With a more recent GEVI, dendritic processes could likely be resolved in single sweeps.

Newer voltage sensors cannot be immediately combined with LFM, however, as they require much faster sampling rates, typically between 500 and $1000 \mathrm{~Hz}$. Megapixel cameras with 1-kHz full-frame readout rates are therefore needed to fully exploit these newer voltage indicators. Current scientific CMOS (sCMOS) cameras such as the one used in this study can achieve these imaging rates by reducing the FOV to a small central strip of the image sensor. This, however, is particularly detrimental to LFM compared to wide-field imaging as the LFM PSF spreads information about each point widely across the image sensor for objects away from the focal plane. If only a small strip of the sensor is imaged, SNR will be greatly degraded as light is lost outside of this reduced FOV. Limiting the camera to 128-pixel rows to achieve a frame rate of $1.6 \mathrm{kHz}$ would reject $<50 \%$ of all fluorescence from objects further than $35 \mu \mathrm{m}$ from the focal plane. This lost light would also be unavailable for the reconstruction, resulting in degraded resolution. This drastically limits the applicability of reducing the FOV for increased frame rate, common with sCMOS cameras.

We anticipate that this issue will be steadily ameliorated as faster sCMOS sensor technology is developed.

A second issue arises with newer, faster GEVIs due to their requirement for much faster frame rates. Deconvolving individual frames with these sensors would require a drastic increase in computational resources and is likely untenable. Source extraction approaches have been developed for static light-field images ${ }^{89}$ and light-field calcium imaging time series ${ }^{65,90}$ which do not involve deconvolution of every frame. In their current form, however, these are unsuitable for reconstruction of subcellular light-field voltage imaging time series as they leverage the temporal and/or spatial characteristics of neuronal calcium imaging as reconstruction priors. These priors, such as somatic signal localization or sparse temporal activity, are not as applicable to subcellular voltage imaging signals, which are smaller, less temporally sparse and arise from more morphologically intricate structures than neuronal somata. Development of more 
sophisticated reconstruction algorithms is nevertheless important, especially as previous efforts have enabled imaging deeper in scattering tissue than was possible in this study.

Finally, in this study, we compared the SNR between refocused LFM volumes and matched wide-field traces and found they did not differ significantly. This is expected, as apart from light losses at the MLA, which are $<15 \%$ according to the manufacturer, there are no significant losses of SNR to shot noise between WFM and LFM. LFM's ability to resolve voltage signals from different axial planes without repeated physical refocusing and time-series acquisition can drastically reduce imaging time and bleaching. Together these results have the potential to motivate further work and widespread application of LFM to voltage imaging owing to light-field's high photon budget and ability to resolve neurons in three spatial dimensions.

\section{Disclosures}

The authors declare that the research was conducted in the absence of any commercial or financial relationships that could be construed as a potential conflict of interest.

\section{Acknowledgments}

We thank Yu Liu for her technical assistance and support for research in the Foust and Schultz labs. The authors would also like to thank the Imperial College Research Computing Service. This work was supported by the following grants: Engineering and Physical Sciences Research Council (Grant No. EP/L016737/1), Wellcome Trust Seed Award (Grant No. 201964/Z/16/Z), the Royal Academy of Engineering under the RAEng Research Fellowships scheme (Grant No. RF1415/14/26), Biotechnology and Biology Research Council (Grant Nos. BB/ R009007/1 and BB/R022437/1), the Royal Society (Grant No. TA/R1/170047), and the BRAIN initiative (U.S. National Institutes of Health, Grant Nos. U01MH109091 and U01NS099573).

\section{Code, Data, and Materials Availability}

The datasets and code generated for this study are available on request to the corresponding author.

\section{References}

1. A. Foust et al., "Action potentials initiate in the axon initial segment and propagate through axon collaterals reliably in cerebellar Purkinje neurons," J. Neurosci. 30(20), 6891-6902 (2010).

2. A. J. Foust et al., "Somatic membrane potential and Kv1 channels control spike repolarization in cortical axon collaterals and presynaptic boutons," J. Neurosci. 31(43), 1549015498 (2011).

3. M. A. Popovic et al., "Cortical dendritic spine heads are not electrically isolated by the spine neck from membrane potential signals in parent dendrites," Cereb. Cortex 24, 385-395 (2014).

4. K. Holthoff, D. Zecevic, and A. Konnerth, "Rapid time course of action potentials in spines and remote dendrites of mouse visual cortex neurons," J. Physiol. 588, 1085-1096 (2010).

5. L. M. Palmer and G. J. Stuart, "Membrane potential changes in dendritic spines during action potentials and synaptic input," J. Neurosci. 29(21), 6897-6903 (2009).

6. D. S. Peterka, H. Takahashi, and R. Yuste, "Imaging voltage in neurons," Neuron 69(1), 9-21 (2011).

7. M. Djurisic et al., "Voltage imaging from dendrites of mitral cells: EPSP attenuation and spike trigger zones," J. Neurosci. 24(30), 6703-6714 (2004).

8. C. J. Roome and B. Kuhn, "Simultaneous dendritic voltage and calcium imaging and somatic recording from Purkinje neurons in awake mice," Nat. Commun. 9(1), 1-14 (2018). 
9. M. Canepari et al., "Imaging inhibitory synaptic potentials using voltage sensitive dyes," Biophys. J. 98(9), 2032-2040 (2010).

10. S. D. Antic, R. M. Empson, and T. Knöpfel, "Voltage imaging to understand connections and functions of neuronal circuits," J. Neurophysiol. 116(1), 135-152 (2016).

11. T. Kwon et al., "Attenuation of synaptic potentials in dendritic spines," Cell Rep. 20(5), 1100-1110 (2017).

12. S. Chamberland et al., "Fast two-photon imaging of subcellular voltage dynamics in neuronal tissue with genetically encoded indicators," Elife 6, e25690 (2017).

13. S. D. Antic, "Action potentials in basal and oblique dendrites of rat neocortical pyramidal neurons," J. Physiol. 550(1), 35-50 (2003).

14. S. Antic, G. Major, and D. Zecevic, "Fast optical recordings of membrane potential changes from dendrites of pyramidal neurons," J. Neurophysiol. 82(3), 1615-1621 (1999).

15. F. Anselmi et al., "Three-dimensional imaging and photostimulation by remote-focusing and holographic light patterning," Proc. Natl. Acad. Sci. U. S. A. 108(49), 19504-19509 (2011).

16. S. Quirin et al., "Calcium imaging of neural circuits with extended depth-of-field light-sheet microscopy," Opt. Lett. 41(5), 855-858 (2016).

17. R. Tomer et al., "Sped light sheet microscopy: fast mapping of biological system structure and function," Cell 163(7), 1796-1806 (2015).

18. K. F. Ahrens et al., "Two-photon scanning microscopy of in vivo sensory responses of cortical neurons genetically encoded with a fluorescent voltage sensor in rat," Front. Neural Circuits 6, 15 (2012).

19. W. Akemann et al., "Two-photon voltage imaging using a genetically encoded voltage indicator," Sci. Rep. 3, 2231 (2013).

20. V. Villette et al., "Ultrafast two-photon imaging of a high-gain voltage indicator in awake behaving mice," Cell 179(7), 1590-1608 (2019).

21. J. Bewersdorf, R. Pick, and S. W. Hell, "Multifocal multiphoton microscopy," Opt. Lett. 23(9), 655-657 (1998).

22. K. H. Kim et al., "Multifocal multiphoton microscopy based on multianode photomultiplier tubes," Opt. Express 15(18), 11658-11678 (2007).

23. T. Nielsen et al., "High efficiency beam splitter for multifocal multiphoton microscopy," J. Microsc. 201(3), 368-376 (2001).

24. Y. Shao et al., "Addressable multiregional and multifocal multiphoton microscopy based on a spatial light modulator," J. Biomed. Opt. 17(3), 030505 (2012).

25. P. Quicke et al, "High speed functional imaging with source localized multifocal two-photon microscopy," Biomed. Opt. Express 9, 3678 (2018).

26. J. Wu et al., "Kilohertz two-photon fluorescence microscopy imaging of neural activity in vivo," Nat. Methods 17(3), 287-290 (2020).

27. T. Zhang et al., "Kilohertz two-photon brain imaging in awake mice," Nat. Methods 16(11), 1119-1122 (2019).

28. C. G. Coates et al., "Optimizing low-light microscopy with back-illuminated electron multiplying charge-coupled device: enhanced sensitivity, speed, and resolution," J. Biomed. Opt. 9(6), 1244-1253 (2004).

29. L. Filipis et al., "A novel multisite confocal system for rapid $\mathrm{Ca}^{2+}$ imaging from submicron structures in brain slices," J. Biophotonics 11(3), e201700197 (2018).

30. R. Prevedel et al., "Fast volumetric calcium imaging across multiple cortical layers using sculpted light," Nat. Methods 13, 1021-1028 (2016).

31. S. Weisenburger et al., "Volumetric $\mathrm{Ca}^{2+}$ imaging in the mouse brain using hybrid multiplexed sculpted light microscopy," Cell 177(4), 1050-1066 (2019).

32. M. Žurauskas et al., "Rapid adaptive remote focusing microscope for sensing of volumetric neural activity," Biomed. Opt. Express 8, 4369 (2017).

33. A. Kazemipour et al., "Kilohertz frame-rate two-photon tomography," Nat. Methods 16(8), 778 (2019).

34. H. Dana et al., "Hybrid multiphoton volumetric functional imaging of large-scale bioengineered neuronal networks," Nat. Commun. 5, 3997 (2014).

35. M. B. Ahrens et al., "Whole-brain functional imaging at cellular resolution using light-sheet microscopy," Nat. Methods 10(5), 413-420 (2013). 
36. J. Huisken et al., "Optical sectioning deep inside live embryos by selective plane illumination microscopy," Science 305(5686), 1007-1009 (2004).

37. P. J. Keller and M. B. Ahrens, "Visualizing whole-brain activity and development at the single-cell level using light-sheet microscopy," Neuron 85(3), 462-483 (2015).

38. M. B. Bouchard et al., "Swept confocally-aligned planar excitation (SCAPE) microscopy for high-speed volumetric imaging of behaving organisms," Nat. Photonics 9, 113-119 (2015).

39. D. Oron, E. Tal, and Y. Silberberg, "Scanningless depth-resolved microscopy," Opt. Express 13(5), 1468-1476 (2005).

40. G. Zhu et al., "Simultaneous spatial and temporal focusing of femtosecond pulses," Opt. Express 13(6), 2153-2159 (2005).

41. T. Schrödel et al., "Brain-wide 3D imaging of neuronal activity in Caenorhabditis elegans with sculpted light," Nat. Methods 10(10), 1013 (2013).

42. C. J. Rowlands et al., "Wide-field three-photon excitation in biological samples," Light: Sci. Appl. 6, e16255 (2017).

43. O. Therrien et al., "Wide-field multiphoton imaging of cellular dynamics in thick tissue by temporal focusing and patterned illumination," Biomed. Opt. Express 2(3), 696-704 (2011).

44. A. J. Foust et al., "Computer-generated holography enhances voltage dye fluorescence discrimination in adjacent neuronal structures," Neurophotonics 2(2), 021007 (2015).

45. V. Nikolenko et al., "SLM microscopy: scanless two-photon imaging and photostimulation using spatial light modulators," Front. Neural Circuits 2, 5 (2008).

46. S. Bovetti et al., "Simultaneous high-speed imaging and optogenetic inhibition in the intact mouse brain," Sci. Rep. 7, 40041 (2017).

47. D. Tanese et al., "Imaging membrane potential changes from dendritic spines using computer-generated holography," Neurophotonics 4(3), 031211 (2017).

48. E. J. Botcherby, R. Juškaitis, and T. Wilson, "Scanning two photon fluorescence microscopy with extended depth of field," Opt. Commun. 268, 253-260 (2006).

49. R. Lu et al., "Video-rate volumetric functional imaging of the brain at synaptic resolution," Nat. Neurosci. 20, 620-628 (2017).

50. A. Song et al., "Volumetric two-photon imaging of neurons using stereoscopy (vTwINS)," Nat. Methods 14(4), 420-426 (2017).

51. M. Levoy et al., "Light field microscopy," in ACM SIGGRAPH 2006 Pap.—SIGGRAPH'06, ACM Press, New York, Vol. 25, No. 3, p. 924 (2006).

52. P. Quicke, "Improved methods for functional neuronal imaging with genetically encoded voltage indicators," PhD Thesis, Imperial College London (2019).

53. M. Broxton et al., "Wave optics theory and 3-D deconvolution for the light field microscope," Opt. Express 21(21), 25418 (2013).

54. W. H. Richardson, "Bayesian-based iterative method of image restoration," J. Opt. Soc. Am. 62, 55 (1972).

55. L. B. Lucy, "An iterative technique for the rectification of observed distributions," Astron. J. 79, 745 (1974).

56. M. E. Daube-Witherspoon and G. Muehllehner, "An iterative image space reconstruction algorithm suitable for volume ECT," IEEE Trans. Med. Imaging 5, 61-66 (1986).

57. H. Li et al., "Fast, volumetric live-cell imaging using high-resolution light-field microscopy," Biomed. Opt. Express 10, 29 (2019).

58. N. Cohen et al., "Enhancing the performance of the light field microscope using wavefront coding," Opt. Express 22(20), 24817-24839 (2014).

59. C. Guo et al., "Fourier light-field microscopy," Opt. Express 27(18), 25573-25594 (2019).

60. L. Cong et al., "Rapid whole brain imaging of neural activity in freely behaving larval zebrafish (Danio rerio)," eLife 6, e28158 (2017).

61. F. L. Liu et al., "Fourier diffuserScope: single-shot 3D Fourier light field microscopy with a diffuser," arXiv:2006.16343 (2020).

62. R. Prevedel et al., "Simultaneous whole-animal 3D imaging of neuronal activity using lightfield microscopy," Nat. Methods 11(7), 727-730 (2014). 
63. N. Wagner et al., "Instantaneous isotropic volumetric imaging of fast biological processes," Nat. Methods 16(6), 497-500 (2019).

64. L. M. Grosenick et al., "identification of cellular-activity dynamics across large tissue volumes in the mammalian brain," bioRxiv, 132688 (2017).

65. T. Nöbauer et al., "Video rate volumetric $\mathrm{Ca}^{2+}$ imaging across cortex using seeded iterative demixing (SID) microscopy," Nat. Methods 14, 811-818 (2017).

66. O. Skocek et al., "High-speed volumetric imaging of neuronal activity in freely moving rodents," Nat. Methods 15, 429-432 (2018).

67. S. Aimon et al., "Fast near-whole-brain imaging in adult Drosophila during responses to stimuli and behavior," PLoS Biol. 17(2), e2006732 (2019).

68. P. Quicke et al., "Single-neuron level one-photon voltage imaging with sparsely targeted genetically encoded voltage indicators," Front. Cell. Neurosci. 13, 39 (2019).

69. C. Song et al., "Transgenic strategies for sparse but strong expression of genetically encoded voltage and calcium indicators," Int. J. Mol. Sci. 18, 1461 (2017).

70. P. Quicke et al., "Calculation of high numerical aperture lightfield microscope point spread functions," in Imaging and Appl. Opt. 2019 (COSI, IS, MATH, pcAOP), OSA, Washington, D.C., Paper CW4A.2 (2019).

71. W. Akemann et al., "Imaging neural circuit dynamics with a voltage-sensitive fluorescent protein,” J. Neurophysiol. 108, 2323-2337 (2012).

72. M. Mayford et al., "Control of memory formation through regulated expression of a CaMKII transgene," Science 274(5293), 1678-1683 (1996).

73. J. A. Harris et al., "Anatomical characterization of Cre driver mice for neural circuit mapping and manipulation," Front. Neural Circuits 8, 76 (2014).

74. L. Madisen et al., "Transgenic mice for intersectional targeting of neural sensors and effectors with high specificity and performance," Neuron 85, 942-958 (2015).

75. J. T. Ting et al., "Acute brain slice methods for adult and aging animals: application of targeted patch clamp analysis and optogenetics.," Methods Mol. Biol. 1183, 221-42 (2014).

76. J. T. Ting, "Improved methods for acute brain slice preparation from adult and aging animals," www.brainslicemethods.com.

77. A. D. Edelstein et al., "Advanced methods of microscope control using $\mu$ Manager software," J. Biol. Methods 1(2), 10 (2014).

78. A. Yaroslavsky et al., "Optical properties of selected native and coagulated human brain tissues in vitro in the visible and near infrared spectral range," Phys. Med. Biol. 47(12), 2059 (2002).

79. P. Virtanen et al., "SciPy 1.0: fundamental algorithms for scientific computing in Python," Nat. Methods 17, 261-272 (2020).

80. R. Ng et al., "Light field photography with a hand-held plenoptic camera," Stanford Tech Report CTSR 2005-02 (2005).

81. E. Thiébaut, "Introduction to image reconstruction and inverse problems," in R. Foy and F. Foy, Eds., Optics in Astrophysics. NATO Science Series II: Mathematics, Physics and Chemistry, Vol. 198, Springer, Dordrecht (2006).

82. N. Dey et al., "Richardson-Lucy algorithm with total variation regularization for 3D confocal microscope deconvolution," Microsc. Res. Tech. 69(4), 260-266 (2006).

83. M. Bertero, P. Boccacci, and V. Ruggiero, Inverse Imaging with Poisson Data, IOP Publishing, Bristol (2018).

84. A. S. Abdelfattah et al., "Bright and photostable chemigenetic indicators for extended in vivo voltage imaging," Science 365(6454), 699-704 (2019).

85. Y. Adam et al., "Voltage imaging and optogenetics reveal behaviour-dependent changes in hippocampal dynamics," Nature 569(7756), 413-417 (2019).

86. K. D. Piatkevich et al., "A robotic multidimensional directed evolution approach applied to fluorescent voltage reporters," Nat. Chem. Biol. 14, 352-360 (2018).

87. B. Yi et al., "A dimeric fluorescent protein yields a bright, red-shifted GEVI capable of population signals in brain slice," Sci. Rep. 8(1), 15199 (2018).

88. M. Kannan et al., "Fast, in vivo voltage imaging using a red fluorescent indicator," Nat. Methods 15(12), 1108-1116 (2018). 
89. P. Song et al., "3D localization for light-field microscopy via convolutional sparse coding on epipolar images," IEEE Trans. Comput. Imaging 6, 1017-1032 (2020).

90. N. C. Pégard et al., "Compressive light-field microscopy for 3D neural activity recording," Optica 3(5), 517-524 (2016).

Peter Quicke is a postdoctoral research associate in the Department of Bioengineering at Imperial College London. He received his MSc degree (with BSc) in physics in 2014, an MRes in neurotechnology in 2015, and his $\mathrm{PhD}$ in 2019. His current research interests include computational microscopy and functional voltage imaging.

Carmel L. Howe is a research associate at Imperial College London, UK. She received her MEng and $\mathrm{PhD}$ degrees in electrical and electronic engineering from the University of Nottingham in 2014 and 2018, respectively. She is currently developing a new high-speed, high-throughput, 3-D imaging modality to track network-level neuronal activity in the mammalian brain. Her research combines the fields of neurophysiology, optical engineering, and signal and image processing.

Pingfan Song is a research associate at Imperial College London. He obtained his $\mathrm{PhD}$ from the University College London, his master's and bachelor's degrees both from Harbin Institute of Technology. His research interests include signal and image processing, machine learning, and computational imaging with applications on a variety of image modalities.

Herman V. Jadan received his bachelor degree in electronic and communications engineering from Escuela Superior Politecnica del Litoral (ESPOL), Guayaquil, Ecuador; the masters degree from Imperial College London, United Kingdom. He is currently working toward the $\mathrm{PhD}$ in the Department of Electrical and Electronic Engineering, Imperial College London. His research interests include sparsity-driven signal/image processing, and machine learning with applications in the solution of inverse problems.

Mark Neil is a professor of photonics in the Department of Physics at Imperial College. He received his BA degree in natural sciences from Cambridge University in 1985 and his $\mathrm{PhD}$ in optical engineering, also from Cambridge, in 1990. His current research interests include optical imaging and microscopy, particularly fluorescence, and super-resolution techniques applied to problems in biology and medicine.

Pier L. Dragotti received his laurea degree (summa cum laude) in electronic engineering from University Federico II, Naples, Italy, in 1997; his master's degree in communications systems from the Swiss Federal Institute of Technology of Lausanne (EPFL), Switzerland, in 1998; and his PhD from EPFL, Switzerland, in April 2002. He has held several visiting positions. In particular, he was a visiting student at Stanford University, Stanford, California, USA, in 1996; a summer researcher at the Mathematics of Communications Department, Bell Labs, Lucent Technologies, Murray Hill, New Jersey, USA, in 2000; and a visiting scientist at the Massachusetts Institute of Technology in 2011. He is currently a professor of signal processing in the Department of Electrical and Electronic Engineering, Imperial College London. Before joining Imperial College in November 2002, he was a senior researcher at EPFL, working on distributed signal processing for sensor networks for the Swiss National Competence Center in Research on Mobile Information and Communication Systems. His research interests include sampling theory, wavelet theory, and its applications, sparsity-driven signal processing with application in image super-resolution, neuroscience, and computational imaging. He was the technical co-chair of the European Signal Processing Conference in 2012, an associate editor of the IEEE Transactions on Image Processing from 2006 to 2009, a member of the IEEE Image, Video and Multidimensional Signal Processing Technical Committee, and a member of the IEEE Signal Processing Theory and Methods Technical Committee. He was also a recipient of the ERC Investigator Award. He is currently the editor-in-chief of the IEEE Transactions on Signal Processing, and a member of the IEEE Computational Imaging Technical Committee and a fellow of IEEE. 
Simon R. Schultz is a professor of neurotechnology and director of the Centre for Neurotechnology at Imperial College London. He received his BSc degree in physics and applied mathematics from Monash University in 1994, his BE degree in electrical and computer engineering also from Monash University in 1994, his ME(Res) degree in electrical engineering from Sydney University in 1997, and his DPhil in neuroscience from Oxford University in 1998. His current research interests include the development of optical technology for understanding brain function.

Amanda J. Foust is a Royal Academy of Engineering Research Fellow and lecturer in the Department of Bioengineering at Imperial College London. She obtained her BSc degree in neuroscience with emphasis in computation and electrical engineering from Washington State University, and MPhil and PhD degrees in neuroscience from Yale University. The aim of her research program is to engineer bridges between cutting-edge optical technologies and neuroscientists to acquire new, groundbreaking data on how brain circuits wire, process, and store information.

Biographies of the other authors are not available. 